\title{
Zabofloxacin versus moxifloxacin in patients with COPD exacerbation: a multicenter, double-blind, double-dummy, randomized, controlled, Phase III, non-inferiority trial
}

\author{
This article was published in the following Dove Press journal: \\ International Journal of COPD \\ 22 October 2015 \\ Number of times this article has been viewed
}

Chin Kook Rhee,' Jung Hyun Chang, ${ }^{2}$ Eu gene Choi, ${ }^{3}$ Hyun kuk Kim, ${ }^{4}$ Yong-Soo Kwon, ${ }^{5}$ Sun Young Kyung, ${ }^{6}$ Ji-Hyun Lee, ${ }^{7}$ Myung Jae Park, ${ }^{8}$ Kwang $\mathrm{Ha}$ Yoo, ${ }^{9}$ Yeon Mok Oh ${ }^{10}$

'Division of Pulmonary, Allergy and Critical Care Medicine, Department of Internal Medicine, Seoul St Mary's Hospital, College of Medicine, The Catholic University of Korea, ${ }^{2}$ Division of Pulmonary and Critical Care Medicine, Department of Internal Medicine, School of Medicine, Ewha Womans University Medical Center, Seoul, ${ }^{3}$ Division of Pulmonary, Allergy and Critical Care Medicine, Department of Internal Medicine, Daejeon Hankook Hospital, Daejeon, ${ }^{4}$ Division of Pulmonary and Critical Care Medicine, Department of Internal Medicine, Haeundae Paik Hospital, Inje University College of Medicine, Busan, ${ }^{5}$ Division of Pulmonary and Critical Care Medicine, Department of Internal Medicine, Chonnam National University Hospital, Gwangju, ${ }^{6}$ Division of Pulmonary, Allergy and Critical Care Medicine, Department of Internal Medicine Gachon University Gil Medical Center, Incheon, ${ }^{7}$ Division of Pulmonary, Allergy and Critical Care Medicine, Department of Internal Medicine, CHA Bundang Medical Center of CHA University, Seongnam, ${ }^{8}$ Division of Pulmonary and Critical Care Medicine, Department of Internal Medicine, KyungHee University Hospital, Seoul,

${ }^{9}$ Division of Pulmonary, Allergy and Critical Care Medicine, Department of Internal Medicine, Konkuk University School of Medicine, ${ }^{10}$ Department of Pulmonary and Critical Care Medicine, Asan Medical Center, University of Ulsan College of Medicine, Seoul, Korea

Correspondence: Yeon Mok Oh

Department of Pulmonary and Critical Care Medicine, Asan Medical Center, University of Ulsan College of Medicine, 88 Olympic-ro 43-gil, Songpa-gu, Seoul 138-736, Korea Tel +82230103136

Fax +82230106968

Email ymoh55@amc.seoul.kr
Abstract: A new quinolone, zabofloxacin, has now been developed; hence, a non-inferiority trial is needed to compare this new compound with another widely used quinolone to examine its efficacy and safety for the treatment of chronic obstructive pulmonary disease (COPD) exacerbations. This was a prospective, multicenter, double-blind, double-dummy, randomized, controlled, parallel-group, Phase III, non-inferiority clinical trial designed to compare oral zabofloxacin ( $367 \mathrm{mg}$ once daily for 5 days) with moxifloxacin (400 $\mathrm{mg}$ once daily for 7 days) for the treatment of patients with COPD exacerbation. In all, 345 COPD patients with a moderate COPD exacerbation were enrolled in the study via the outpatient clinics at 31 university hospitals. Clinical per protocol analysis revealed that the clinical cure rate for zabofloxacin was $86.7 \%$ and that for moxifloxacin was $86.3 \%$ (the rate difference, $0.4 \%$; $95 \%$ confidence interval, $-7.7 \%-8.6 \%$ ). Intention-to-treat analysis revealed clinical cure rates of $77.1 \%$ and $77.3 \%$ (difference, $-0.2 \% ; 95 \%$ confidence interval, $-9.0 \%-8.8 \%$ ), respectively. These results confirm that zabofloxacin is not inferior to moxifloxacin. The favorable microbiological response rate for zabofloxacin was $67.4 \%$ and that for moxifloxacin was $79.5 \%(P=0.22)$. Patients in the zabofloxacin group showed better patient-oriented outcomes, as measured by EXAcerbations of Chronic Pulmonary Disease Tool-Patient-Reported Outcome and the COPD assessment test scores, than patients in the moxifloxacin group. Adverse drug reactions related to zabofloxacin occurred in $9.7 \%$ of cases and those related to moxifloxacin occurred in $9.6 \%$ of cases $(P=0.97)$. The dropout rate due to adverse events was $0 \%(0 / 175)$ in the zabofloxacin group and $1.8 \%$ $(3 / 167)$ in the moxifloxacin group $(P=0.12)$. Oral zabofloxacin ( $367 \mathrm{mg}$ once daily for 5 days) was not inferior to oral moxifloxacin ( $400 \mathrm{mg}$ once daily for 7 days) for the treatment of patients with COPD exacerbation.

Keywords: chronic obstructive pulmonary disease, exacerbation, zabofloxacin, quinolone, EXACT-PRO, CAT

\section{Introduction}

Exacerbation of chronic obstructive pulmonary disease (COPD) is characterized by a worsening of respiratory symptoms. The condition is important because it can negatively affect the quality of life of COPD patients. Moreover, exacerbation can be associated with significant mortality. Thus, adequate treatment of COPD exacerbation is mandatory. Most incidences of exacerbation are caused by respiratory tract infections; ${ }^{1,2}$ thus, antibiotics are a key component of any treatment regimen. Antibiotics are indicated when there is evidence of an increase in either the purulence or volume of sputum. ${ }^{3}$ 
Zabofloxacin is a new fluoroquinolone antibiotic. Previous studies show that zabofloxacin has excellent activity against both Gram-negative and Gram-positive pathogens; ${ }^{4-6}$ however, no study has examined its effect on COPD exacerbation. Other fluoroquinolone antibiotics such as levofloxacin ${ }^{7}$ and moxifloxacin ${ }^{8}$ show good efficacy and safety profiles when used to treat patients with COPD exacerbation.

Therefore, the aim of the present study was to compare the efficacy and safety of oral zabofloxacin (367 mg once daily for 5 days) with that of moxifloxacin (400 $\mathrm{mg}$ once daily for 7 days) for the treatment of patients with COPD exacerbation.

\section{Materials and methods Study design}

This was a prospective, multicenter, double-blind, doubledummy, randomized, controlled, parallel-group, Phase III, non-inferiority clinical trial designed to compare oral zabofloxacin (367 mg once daily for 5 days) with moxifloxacin (400 mg once daily for 7 days) for the treatment of patients with COPD exacerbation. The study was conducted between September 2012 and February 2014. This study was approved by all relevant ethical committees at each participating institution.

\section{Study subjects}

The enrollment criteria were as follows: 1) patients with COPD; 2 ) age $\geq 40$ years; 3 ) patients experiencing moderate (patients who did not need hospitalization) exacerbation of COPD (worsening of the respiratory symptoms that is beyond normal day-to-day variations and leads to a change in medication); 4) a post-bronchodilator forced expiratory volume in 1 second/forced vital capacity $<0.7$; and 5) purulent sputum or increased volume of sputum. The exclusion criteria were as follows: 1) pregnant women; 2) patients who received systemic antibiotics and/or antifungal agents within the last 72 hours; 3 ) confirmed pneumonia (on chest X-ray) within 48 hours; 4) patients with underlying septic shock, bronchiectasis, lung abscess, active tuberculosis, pulmonary malignancy, cystic fibrosis, empyema, or asthma; 5) patients with kidney or liver disease with abnormal laboratory test results (creatinine clearance $<50 \mathrm{~mL} / \mathrm{min} / 1.73 \mathrm{~m}^{2}$; blood urea nitrogen $>30 \mathrm{mg} / \mathrm{dL}$; aspartate aminotransferase or alanine aminotransferase $>3$ times the upper limit of normal; total bilirubin $>2$ times upper limit of normal; or alkaline phosphatase $>2$ times upper limit of normal); 6) patients with an organic gastrointestinal disorder (such as active Crohn's disease or active ulcerative colitis) within 6 months; 7) an absolute neutrophil count $<1,000$ cells $/ \mathrm{mm}^{3}$; 8) patients with chronic hepatitis B or C; 9) immunocompromised patients (human immunodeficiency virus-positive, acquired immune deficiency syndrome, bone marrow transplantation, or leukemia); 10) a history of hypersensitivity to fluoroquinolone antibiotics; 11) a history of seizure or anti-seizure medications; 12) a history of ventricular arrhythmia; and 13) a history of corrected QT prolongation or treatment with medication that prolongs the corrected QT interval.

\section{Randomization}

Patients were randomized on a 1:1 basis and stratified according to the presence of chronic bronchitis (presence of cough and sputum production for at least 3 months in two consecutive years). Subjects were allocated via a blocked randomization protocol that was prepared beforehand; the ratio for each group was 1:1. The randomization list was applied independently via random numbers, which were generated by the randomization program within the SAS System. Numbers were generated according to the order in which the subjects were enrolled by each investigational institution. The randomization list was derived prior to the clinical study by a statistician employed by a contract research organization specified by the study sponsor.

\section{Investigational drugs}

The test drug was manufactured by Dongwha Pharm. Co., Ltd. (Chungju City, Seoul, Korea). The placebo for the test drug was also manufactured by Dongwha Pharm. Co., Ltd. The placebo preparation was identical to that of the drug but lacked the active ingredient, zabofloxacin D-aspartate. The comparator, Avelox ${ }^{\circledR} 400$ mg (Bayer Korea Ltd., Seoul, Korea), was purchased from Bayer Korea Ltd. The placebo for the comparator was manufactured by NextPharma Allphamed PHARBIL Arzneimittel GmbH (Göttingen, Germany). Again, the placebo lacked the active ingredient, moxifloxacin hydrochloride. The test drug, the test drug placebo, the comparator, and the comparator placebo were packaged by Dongwha Pharm. Co., Ltd. in accordance with a previously-prepared randomization list. The drugs were then kept in the investigational products storage facility.

\section{Blinding}

The test drug, comparator, and placebos were indistinguishable in terms of appearance. The allocation information was blinded from all site investigators and subjects.

\section{Study protocol}

Patients were randomized to receive oral zabofloxacin $(367 \mathrm{mg}$ once daily for 5 days) and placebo (once daily for 2 days), 
or moxifloxacin (400 mg once daily for 7 days) during seven consecutive days. History taking, physical examination, laboratory tests, an electrocardiogram, and a chest X-ray were performed on Day 1 (inclusion visit, visit 1). Sputum samples were also collected. Patients who met the enrollment criteria and provided written informed consent were randomized and given the drugs. Patients were assessed over the telephone on treatment Day $4 \pm 1$ (visit 2). Clinical response, microbiological response, and drug safety were assessed on the test of cure (TOC) visit on Day $10 \pm 3$ (visit 3). These parameters were reassessed at the end of study (EOS) visit on Day $36 \pm 7$ (visit 4).

\section{Clinical assessment}

Clinical failure was defined as a lack of improvement in dyspnea or sputum purulence/volume at TOC compared with the inclusion visit coupled with a requirement for additional antibiotic therapy. Clinical cure was defined as an improvement in dyspnea or sputum purulence/volume at TOC compared with the inclusion visit. Clinical response, microbiological response, and safety were assessed again at the EOS visit. Relapse was defined as a worsening of dyspnea or sputum purulence compared with the TOC visit (ie, the patient met the definition of "clinical cure" at the TOC visit but required additional antibiotic therapy between the TOC and EOS visits).

\section{Microbiological assessment}

Microbiological responses were classified as follows: eradication, presumed eradication, persistence, presumed persistence, recurrence, indeterminate, and emergent infection. Eradication and presumed eradication were favorable microbiological responses whereas persistence, presumed persistence, recurrence, indeterminate, and emergent infection were unfavorable microbiological responses. Eradication was defined as confirmed pathogen-free after the initial pathogen had been identified. The definition of presumed eradication was that additional samples were either uncollectable or non-culturable after the initial pathogen was identified and there were no clinical signs of persistent infection. Persistence was defined as the detection of pathogen after the initial pathogen was identified. The definition of presumed persistence was that additional samples were either uncollectable or non-culturable after the initial pathogen had been identified and there were clinical signs of persistent infection. Recurrence was defined as detection of the same pathogen (initially confirmed) at the EOS visit, even though the patient had been classified into the eradication or presumed eradication groups at the
TOC visit. Indeterminate response was defined as the lack of a confirmed pathogen coupled with an unclear clinical response. Emergent infection was defined as the detection of any pathogen (other than the initial pathogen) in a patient showing clinical signs of infection.

\section{Symptom scores}

Symptom severity was determined by examining the EXAcerbations of Chronic Pulmonary Disease Tool (EXACT)Patient-Reported Outcome (PRO) and COPD assessment test (CAT) scores obtained on Days 1-7 and on Day 36.

\section{Primary and secondary endpoints}

The primary endpoint was the clinical response rate at the time of the TOC visit in the per protocol (PP) group. The secondary endpoints were as follows: 1) clinical response rate at the EOS visit; 2) clinical response rate in the microbiological PP group at the TOC and EOS visits; 3) microbiological response rate in the microbiological $\mathrm{PP}$ group at the TOC and EOS visits; 4) changes in the EXACT-PRO score at the TOC and EOS visits; and 5) changes in the CAT score at the TOC and EOS visits.

\section{Safety assessments}

Drug safety was assessed in all study subjects who received at least one dose of the investigational product. Data related to adverse events, vital signs, and laboratory tests, as well as findings from physical examinations, were reviewed. All collected data were evaluated in terms of severity, changes from baseline values, and relationships to treatment with the investigational products.

\section{Statistical analysis}

All statistical analyses were performed using SAS version 9.2 (SAS Institute, Inc., Cary, NC, USA). The primary aim of the study was to reject the null hypothesis that zabofloxacin therapy was $>10 \%$ less effective than moxifloxacin therapy. To examine the difference in the clinical cure rate, the $95 \%$ confidence interval (CI) for zabofloxacin minus that of moxifloxacin was calculated using Mantel-Haenszel weights. For zabofloxacin to be considered no less effective than moxifloxacin, the lower limit of this CI had to be greater than $-10 \%$. The clinical cure rate of moxifloxacin in a previous study was $91 \%{ }^{8}$ and the clinical cure rate of the test drug and the comparator were presumed as $91 \%$. Level of significance $(\alpha)$ was 0.05 and type 2 error $(\beta)$ was set as 0.2 to maintain the power of the test as $80 \%$. The number of cases required for the clinical trial was 129 subjects per group. Dropout rate was expected as $25 \%$ and 
calculated number of subjects in each group was 172 . Both intention-to-treat (ITT) and PP analyses were performed. Differences between groups were assessed using the chisquared test or Fisher's exact test (categorical variables) and Student's $t$-test or the Mann-Whitney $U$-test (continuous variables). A paired $t$-test was used to compare changes in the EXACT-PRO and CAT scores between Day 1 and the other test days. To compare changes in the EXACT-PRO and CAT scores between the zabofloxacin- and moxifloxacin-treated groups, the delta values (differences between score on Day 1 and other test days) for each group were compared using Student's $t$-test. Data are expressed as the mean \pm standard deviation throughout the paper except in the figures. Data are expressed as mean \pm standard error of mean in figures.

This study is registered with ClinicalTrials.gov number NCT01658020 and Clinical Research Information Service registry number KCT0000532.

\section{Results}

\section{Study subjects}

A total of 345 COPD patients with moderate exacerbation were enrolled in the study via the outpatient clinics at 31 university hospitals. Patients were randomly assigned to receive zabofloxacin $(n=176)$ or moxifloxacin $(n=169)$. In all, 342 subjects (175 zabofloxacin and 167 moxifloxacin) were included in the ITT analysis (three patients were excluded in the ITT because the drug was not administered). A total of 274 subjects (143 zabofloxacin and 131 moxifloxacin) were included in the clinical PP analysis and 82 subjects (43 zabofloxacin and 39 moxifloxacin) were included in the microbiological PP analysis (Figure 1).

Baseline characteristics are described in Table 1. There were no significant differences in the baseline characteristic of the two groups, except sex and smoking history.

\section{Clinical response}

The clinical cure rates in the PP analysis at the time of the TOC visit were $86.7 \%$ (124/143) for zabofloxacin and $86.3 \%$ $(113 / 131)$ for moxifloxacin. The difference in the clinical cure rate between the two antibiotics (zabofloxacin minus moxifloxacin) was $0.4 \%(95 \% \mathrm{CI},-7.7 \%-8.6 \%)$. ITT analysis revealed the clinical cure rates to be $77.1 \%(135 / 175)$ and $77.3 \%$ (129/167), respectively, with a difference of $-0.1 \%$ (95\% CI, $-9.0 \%-8.8 \%$ ) (Table 2). These results confirm that zabofloxacin was not inferior to moxifloxacin in terms of clinical efficacy at TOC. Clinical PP analysis showed that the clinical cure rate for zabofloxacin at the time of the EOS visit was $76.2 \%$ (109/143), whereas that for moxifloxacin was
$71.0 \%(93 / 131, P=0.28)$. Clinical ITT analysis showed that the clinical cure rate for zabofloxacin at the time of the EOS visit was $68.6 \%(120 / 175)$, whereas that for moxifloxacin was $64.7 \%(108 / 167, P=0.67)$.

Clinical PP analysis of the subgroup of patients with chronic bronchitis showed that at the time of the TOC visit, the clinical cure rate for zabofloxacin was $88.2 \%(45 / 51)$ and that for moxifloxacin was $89.1 \%(49 / 55, P=0.89)$. The clinical cure rates for the subgroup of patients without chronic bronchitis were $85.9 \%(79 / 82)$ and $84.2 \%(64 / 76)$, respectively $(P=0.76)$. ITT analysis showed that the clinical cure rates for the subgroup of patients with chronic bronchitis were $80 \%(52 / 65)$ and $77.5 \%(55 / 71, P=0.64)$, respectively, and those for the subgroup of patients without chronic bronchitis were $75.5 \%(83 / 110)$ and $77.1 \%(74 / 96)$, respectively $(P=0.87)$.

\section{Microbiological response}

The antimicrobial susceptibilities of pathogens isolated at visit 1 are described in Table 3. Microbiological PP analysis of the microbiological response rates at the TOC visit revealed that 29 subjects $(67.4 \%)$ in the zabofloxacin group and 31 subjects $(79.5 \%)$ in the moxifloxacin group presented with a favorable microbiological response (eradication or presumed eradication) $(P=0.22)$. Microbiological PP analysis at EOS revealed that 27 subjects $(62.8 \%)$ in the zabofloxacin group and 25 subjects $(64.1 \%)$ in the moxifloxacin group showed a favorable microbiological response ( $P=0.90$, Table 4).

\section{Changes in the CAT and EXACT-PRO scores}

Clinical PP analysis revealed that the EXACT-PRO scores on Days 2, 3, 4, 5, 6, 7, and 36 decreased significantly when compared with the scores on Day 1 for both the zabofloxacin and moxifloxacin groups (Figure 2A). However, the decline of EXACT-PRO scores for the zabofloxacin group on Days 6 and 7 were significantly greater than those of the moxifloxacin group (Figure 2C). PP analysis showed that the CAT scores on Days 3, 4, 5, 6, 7, and 36 were significantly lower than those on Day 1 for both the zabofloxacin and moxifloxacin groups (Figure 2B). Again, the decline in the CAT scores on Days 5, 6, and 7 was significantly greater in the zabofloxacin than moxifloxacin groups (Figure 2D).

Clinical ITT analysis of the EXACT-PRO scores showed that the scores on Days 2, 3, 4, 5, 6, 7, and 36 were significantly lower than those on Day 1 for both the zabofloxacin and moxifloxacin groups (Figure 3A). However, the decline 


\begin{tabular}{|c|c|c|c|c|c|c|}
\hline \multicolumn{3}{|c|}{ Assessed for eligibility $(n=428)$} & & & & \\
\hline & & & \multicolumn{4}{|c|}{ Screening failure $(n=83)$} \\
\hline & & & \multicolumn{3}{|c|}{$\begin{array}{l}\text { Not meeting inclusion and/or exclusion criteria } \\
\text { Failure to collect sputum at visit } 1 \text { (day } 1) \\
\text { Subject consent withdrawal }\end{array}$} & $\begin{array}{l}(n=80) \\
(n=2) \\
(n=1)\end{array}$ \\
\hline \multicolumn{3}{|c|}{ Randomized $(n=345)$} & & & & \\
\hline & & & \multicolumn{4}{|c|}{ Excluded clinical ITT set $(n=3)$} \\
\hline & & & \multicolumn{3}{|l|}{ Investigational product not taken } & $(n=3)$ \\
\hline \multicolumn{3}{|c|}{ Clinical ITT set ${ }^{1}(n=342)$} & & & & \\
\hline \multirow{2}{*}{\multicolumn{2}{|c|}{$\begin{array}{l}\text { Allocated to } \\
\text { zabofloxacin } \\
(n=175)\end{array}$}} & $\begin{array}{l}\text { Allocated to } \\
\text { moxifloxacin } \\
(n=167)\end{array}$ & & & & \\
\hline & & & Excluded clinical PP set & $\begin{array}{l}\text { Total } \\
n=68\end{array}$ & $\begin{array}{l}\text { ZBO } \\
n=32\end{array}$ & $\begin{array}{l}\text { MOX } \\
n=36\end{array}$ \\
\hline \multicolumn{3}{|c|}{ Number of patients at each visit } & Concomitant medication violation & $n=68$ & $n=13$ & $\mathrm{n}=12$ \\
\hline Visit & ZBO & MOX & Protocol violation & $n=24$ & $\mathrm{n}=11$ & $\mathrm{n}=13$ \\
\hline Visit 1 & $\mathrm{n}=175$ & $\mathrm{n}=167$ & Non-compliance administration & $n=7$ & $\mathrm{n}=2$ & $\mathrm{n}=5$ \\
\hline Visit 2 & $\mathrm{n}=173$ & $n=165$ & Subject consent withdrawal & $\mathrm{n}=6$ & $\mathrm{n}=3$ & $\mathrm{n}=3$ \\
\hline Visit 3 & $\mathrm{n}=170$ & $n=160$ & Adverse events & $\mathrm{n}=5$ & $\mathrm{n}=2$ & $\mathrm{n}=3$ \\
\hline Visit 4 & $\mathrm{n}=171$ & $n=162$ & Lost to follow-up & $n=1$ & $\mathrm{n}=1$ & $\mathrm{n}=0$ \\
\hline
\end{tabular}

\begin{tabular}{|c|c|c|c|c|c|}
\hline \multicolumn{2}{|c|}{ Clinical PP set ${ }^{2}(n=274)$} & & & & \\
\hline $\begin{array}{l}\text { Analyzed to } \\
\text { zabofloxacin } \\
(n=143)\end{array}$ & $\begin{array}{l}\text { Analyzed to } \\
\text { moxifloxacin } \\
(n=131)\end{array}$ & & & & \\
\hline & & Excluded microbiological PP set & Total & ZBO & MOX \\
\hline & & Inability of pathogen separation & $n=192$ & $n=100$ & $n=92$ \\
\hline
\end{tabular}

\begin{tabular}{|l|l|}
\hline \multicolumn{2}{|c|}{ Microbiological PP set ${ }^{3}$} \\
\hline $\begin{array}{l}\text { Analyzed to } \\
\text { zabofloxacin } \\
(n=43)\end{array}$ & $\begin{array}{l}\text { Analyzed to } \\
\text { moxifloxacin } \\
(n=39)\end{array}$ \\
\hline
\end{tabular}

Figure I Disposition of patients during study.

Notes: 'Clinical ITT set: This population consisted of all randomized subjects who received at least one dose of the investigational product. ${ }^{2}$ Clinical PP set: This population consisted of all randomized subjects who met the study inclusion and/or exclusion criteria, who received an adequate course of therapy, for whom sufficient information was available to determine the subject's outcome, and who had no confounding factors that interfered with the assessment of the outcome. ${ }^{3}$ Microbiological PP set: This population consisted of a subset of the clinical PP subjects who had a baseline pathogen identified.

Abbreviations: ZBO, zabofloxacin; MOX, moxifloxacin; ITT, intention-to-treat; PP, per protocol.

in the scores for the zabofloxacin group on Days 6 and 7 was significantly greater than those for the moxifloxacin group (Figure 3C). Clinical ITT analysis of the CAT scores showed that the scores for the zabofloxacin group on Days $2,3,4,5,6,7$, and 36 were significantly lower than those on Day 1; however, the scores for the moxifloxacin group were significantly lower on Days 3, 4, 5, 6, 7, and 36 than on Day 1 (Figure 3B). The decline in the CAT scores for the zabofloxacin group on Days 5, 6, 7, and 36 was significantly greater than that in the moxifloxacin group (Figure 3D). 
Table I Baseline characteristics of the patients

\begin{tabular}{|c|c|c|c|}
\hline Characteristics & $\begin{array}{l}\text { Zabofloxacin } \\
(n=175)(\%)\end{array}$ & $\begin{array}{l}\text { Moxifloxacin } \\
(n=167)(\%)\end{array}$ & $P$-value \\
\hline Age, years & $67.8 \pm 7.8$ & $68.4 \pm 8.0$ & 0.46 \\
\hline Sex, male & $153(87.4)$ & $159(95.2)$ & 0.01 \\
\hline Height, cm & $162.9 \pm 6.5$ & $164.1 \pm 6.3$ & 0.10 \\
\hline Weight, kg & $59.8 \pm 11.1$ & $58.7 \pm 9.6$ & 0.33 \\
\hline Smoking history & & & $<0.01$ \\
\hline Current & $50(28.6)$ & $38(22.8)$ & \\
\hline Ex & $107(61.1)$ & $126(75.5)$ & \\
\hline Never & $18(10.3)$ & $3(1.8)$ & \\
\hline Alcohol & & & 0.29 \\
\hline Never & $26(14.9)$ & $21(12.6)$ & \\
\hline Ex & $91(52.0)$ & $101(60.5)$ & \\
\hline Current & $58(33.1)$ & $45(27.0)$ & \\
\hline Chronic bronchitis & $65(37.1 \%)$ & 7I (42.5\%) & 0.31 \\
\hline \multicolumn{4}{|l|}{ PFT (post-BD) } \\
\hline $\mathrm{FVC} \mathrm{(L)}$ & $2.9 \pm 0.8$ & $3.1 \pm 0.7$ & 0.10 \\
\hline FVC (\%) & $74.9 \pm 17.0$ & $76.5 \pm 16.7$ & 0.40 \\
\hline $\mathrm{FEV}_{1}(\mathrm{~L})$ & $1.4 \pm 0.5$ & $1.4 \pm 0.5$ & 0.93 \\
\hline $\mathrm{FEV}_{1}(\%)$ & $50.5 \pm 18.1$ & $49.1 \pm 17.2$ & 0.51 \\
\hline $\mathrm{FEV}_{\mathrm{I}} / \mathrm{FVC}(\%)$ & $47.8 \pm 12.7$ & $45.2 \pm 12.6$ & 0.07 \\
\hline Dyspnea & & & 0.60 \\
\hline Mild & $27(15.4)$ & $25(15.0)$ & \\
\hline Moderate & $113(64.6)$ & $101(60.5)$ & \\
\hline Severe & $35(20.0)$ & $4 I(24.6)$ & \\
\hline \multicolumn{4}{|l|}{ Comorbidity } \\
\hline Ischemic heart disease & I3 (7.4) & $14(8.4)$ & 0.74 \\
\hline Congestive heart failure & $12(6.9)$ & $7(4.2)$ & 0.28 \\
\hline Cerebrovascular disease & $7(4.0)$ & $3(1.8)$ & 0.34 \\
\hline Diabetes mellitus & $15(8.6)$ & $18(10.8)$ & 0.49 \\
\hline Hypertension & $50(28.6)$ & $48(28.7)$ & 0.97 \\
\hline Osteoporosis & II (6.3) & $6(3.6)$ & 0.25 \\
\hline Hyperlipidemia & I4 (8.0) & $9(5.4)$ & 0.34 \\
\hline \multicolumn{4}{|l|}{ Concomitant medication } \\
\hline $\begin{array}{l}\text { Systemic steroid during } \\
\text { exacerbation }\end{array}$ & $81(46.3)$ & $85(50.9)$ & 0.39 \\
\hline LABA & $17(9.7)$ & $28(16.8)$ & 0.05 \\
\hline LAMA & $116(66.3)$ & $118(70.7)$ & 0.38 \\
\hline ICS & $9(5.1)$ & $9(5.4)$ & 0.92 \\
\hline$I C S+L A B A$ & $99(56.6)$ & $89(53.3)$ & 0.54 \\
\hline Theophylline & $29(16.6)$ & $2 I(12.6)$ & 0.30 \\
\hline PDE4I & $9(5.1)$ & $12(7.2)$ & 0.43 \\
\hline \multicolumn{4}{|l|}{ Symptom score on Day I } \\
\hline EXACT-PRO & $45.5 \pm 9.6$ & $46.7 \pm 11.9$ & 0.31 \\
\hline CAT & $22.6 \pm 7.0$ & $23.2 \pm 7.6$ & 0.45 \\
\hline
\end{tabular}

Note: Data are expressed as mean \pm standard deviation or as number (percentage) of patients.

Abbreviations: PFT, pulmonary function test; BD, bronchodilator; FVC, forced vital capacity; FEV , forced expiratory volume in I second; LABA, long-acting beta agonist; LAMA, long-acting muscarinic antagonist; ICS, inhaled corticosteroid; PDE4I, phosphodiesterase 4 inhibitor; EXACT-PRO, EXAcerbations of Chronic Pulmonary Disease Tool-Patient-Reported Outcome; CAT, chronic obstructive pulmonary disease assessment test.

\section{Adverse events}

Adverse events occurred in 36.6\% (64/175; 108 events) of patients in the zabofloxacin group and in $38.9 \%(65 / 167$; 105 events) of patients in the moxifloxacin group $(P=0.65)$.
The rate of adverse drug reactions to zabofloxacin was $9.7 \%(17 / 175 ; 25$ events $)$ and that to moxifloxacin was $9.6 \%$ (16/167; 23 events); the difference was not statistically significant $(P=0.97$, Table 5$)$. Serious adverse events occurred in 4\% (7/175; seven events: COPD exacerbation in four cases, influenza like illness in one, pneumonia in one, acute pyelonephritis in one) of patients in the zabofloxacin group and in 4.8\% (8/167; eight events: COPD exacerbation in four cases, pneumonia in two, variant angina in one, urethral stenosis in one) of patients in the moxifloxacin group; again, the difference was not statistically significant $(P=0.72)$. There was no incidence of serious adverse drug reaction. The dropout rate due to adverse events was $0 \%$ $(0 / 175)$ in the zabofloxacin group and $1.8 \%(3 / 167)$ in the moxifloxacin group $(P=0.12)$.

\section{Discussion}

The present study compared the efficacy and safety of zabofloxacin with that of moxifloxacin for the treatment of COPD patients with exacerbation. Both drugs showed comparable efficacy and a good safety profile. Also, there were no significant differences in the microbiological response rates. Interestingly, analysis of the EXACT-PRO and CAT scores revealed that zabofloxacin seemed to be superior to moxifloxacin.

There have been several randomized clinical trials to compare the effect of moxifloxacin with other antibiotics on exacerbation of chronic bronchitis. Moxifloxacin was not inferior to clarithromycin, ${ }^{9,10}$ azithromycin, ${ }^{11,12}$ ceftriaxone, ${ }^{13}$ and amoxicillin/clavulanic acid. ${ }^{14}$ In a meta-analysis, ${ }^{15}$ moxifloxacin was clinically equivalent and bacteriologically superior to the antibiotic regimens routinely used in patients with exacerbation of chronic bronchitis. This comparison was made between moxifloxacin and non-quinolone antibiotics.

Urueta-Robledo et $\mathrm{al}^{8}$ showed that a 5 -day course of moxifloxacin was clinically and bacteriologically equivalent to a 7-day course of levofloxacin for the treatment of patients with an acute exacerbation of chronic bronchitis. Here, we found that a 5-day course of zabofloxacin was clinically and bacteriologically equivalent to a 7-day course of moxifloxacin for the treatment of patients with an acute exacerbation of COPD. Because levofloxacin, moxifloxacin, and zabofloxacin are all quinolone antibiotics, the clinical efficacy may be similar. The main purpose of this study is to prove the non-inferiority of zabofloxacin to moxifloxacin. However, we also aimed to see whether a shorter period of administration of zabofloxacin results in comparable clinical 
Table 2 Clinical response rates

\begin{tabular}{|c|c|c|c|c|}
\hline & \multicolumn{2}{|l|}{ Clinical PP } & \multicolumn{2}{|l|}{ Clinical ITT } \\
\hline & $\begin{array}{l}\text { Zabofloxacin } \\
(n=\mid 43)(\%)\end{array}$ & $\begin{array}{l}\text { Moxifloxacin } \\
(n=131)(\%)\end{array}$ & $\begin{array}{l}\text { Zabofloxacin } \\
(n=175)(\%)\end{array}$ & $\begin{array}{l}\text { Moxifloxacin } \\
(n=167)(\%)\end{array}$ \\
\hline \multicolumn{5}{|l|}{ Test of cure } \\
\hline Clinical cure & $124(86.7)$ & $113(86.3)$ & I $35(77.1)$ & $129(77.3)$ \\
\hline $95 \% \mathrm{Cl}$ of clinical cure & $(81.2,92.3)$ & $(80.4,92.2)$ & $(71.9,84.2)$ & $(72.9,85.4)$ \\
\hline Clinical failure & $19(13.3)$ & $18(13.7)$ & $20(11.4)$ & $18(10.8)$ \\
\hline Indeterminate & $0(0.0)$ & $0(0.0)$ & $20(11.4)$ & $20(12.0)$ \\
\hline \multicolumn{5}{|l|}{ End of study } \\
\hline Clinical cure & $109(76.2)$ & $93(71.0)$ & $120(68.6)$ & $108(64.7)$ \\
\hline $95 \% \mathrm{Cl}$ of clinical cure & $(69.3,83.2)$ & $(63.2,78.8)$ & $(62.5,76.2)$ & $(59.0,73.5)$ \\
\hline Clinical failure & $33(23.1)$ & $34(26.0)$ & $34(19.4)$ & $34(20.4)$ \\
\hline Relapse & $14(9.8)$ & $16(12.2)$ & $14(8.0)$ & $16(9.6)$ \\
\hline Indeterminate & I (0.7) & $4(3.1)$ & $21(12.0)$ & $25(15.0)$ \\
\hline
\end{tabular}

Abbreviations: PP, per protocol; ITT, intention-to-treat; $\mathrm{Cl}$, confidence interval.

Table 3 Antimicrobial susceptibility of pathogens isolated at visit I (enrollment day)

\begin{tabular}{|c|c|c|c|c|c|c|c|}
\hline \multirow[t]{2}{*}{ Pathogen } & \multirow[t]{2}{*}{$n$} & \multicolumn{3}{|c|}{ Zabofloxacin } & \multicolumn{3}{|c|}{ Moxifloxacin } \\
\hline & & $\mathrm{MIC}_{50}$ & $\mathrm{MIC}_{90}$ & Range & $\mathrm{MIC}_{50}$ & $\mathrm{MIC}_{90}$ & Range \\
\hline Haemophilus influenzae & 54 & 0.004 & 0.008 & $0.002-0.25$ & 0.016 & 0.016 & $0.004-0.5$ \\
\hline Pseudomonas aeruginosa & 28 & I & 8 & $\leq 0.125-32$ & 2 & 16 & $\leq 0.125-64$ \\
\hline Streptococcus pneumoniae & 27 & 0.031 & 0.125 & $0.008-0.25$ & 0.25 & 1 & $0.125->2$ \\
\hline Moraxella catarrhalis & 16 & 0.016 & 0.016 & $0.004-0.016$ & 0.063 & 0.063 & $0.016-0.063$ \\
\hline
\end{tabular}

Notes: $\mathrm{n}$, number of patients with pathogen in sputum. $\mathrm{MIC}_{50}, \mathrm{MIC}_{90}$, and range values are expressed as $\mu \mathrm{g} / \mathrm{mL}$.

Abbreviation: MIC, minimum inhibitory concentration.

Table 4 Microbiological response according to the microbiological per protocol analysis

\begin{tabular}{|c|c|c|c|}
\hline & $\begin{array}{l}\text { Zabofloxacin } \\
n=43(\%)\end{array}$ & $\begin{array}{l}\text { Moxifloxacin } \\
n=39(\%)\end{array}$ & $P$-value \\
\hline Test of cure & & & 0.22 \\
\hline Favorable bacteriological response & $29(67.4)$ & $31(79.5)$ & \\
\hline Eradication & II (25.6) & II (28.2) & \\
\hline Presumed eradication & $18(4 \mid .9)$ & $20(5 \mid .3)$ & \\
\hline Unfavorable bacteriological response & $14(32.6)$ & $8(20.5)$ & \\
\hline Persistence & II (25.6) & $5(12.8)$ & \\
\hline Presumed persistence & $\mathrm{I}(2.3)$ & $0(0.0)$ & \\
\hline Recurrence & $0(0.0)$ & $0(0.0)$ & \\
\hline Indeterminate & $0(0.0)$ & $0(0.0)$ & \\
\hline Emergent infection & $2(4.7)$ & $3(7.7)$ & \\
\hline End of study & & & 0.90 \\
\hline Favorable bacteriological response & $27(62.8)$ & $25(64.1)$ & \\
\hline Eradication & $10(23.3)$ & $8(20.5)$ & \\
\hline Presumed eradication & $17(39.5)$ & $17(43.6)$ & \\
\hline Unfavorable bacteriological response & $16(37.2)$ & $14(35.9)$ & \\
\hline Persistence & $5(11.6)$ & $3(7.7)$ & \\
\hline Presumed persistence & $2(4.7)$ & $2(5.1)$ & \\
\hline Recurrence & $4(9.3)$ & $2(5.1)$ & \\
\hline Indeterminate & $0(0.0)$ & $0(0.0)$ & \\
\hline Emergent infection & $5(11.6)$ & $7(18.0)$ & \\
\hline
\end{tabular}

Abbreviation: $\mathrm{n}$, number of patients. 

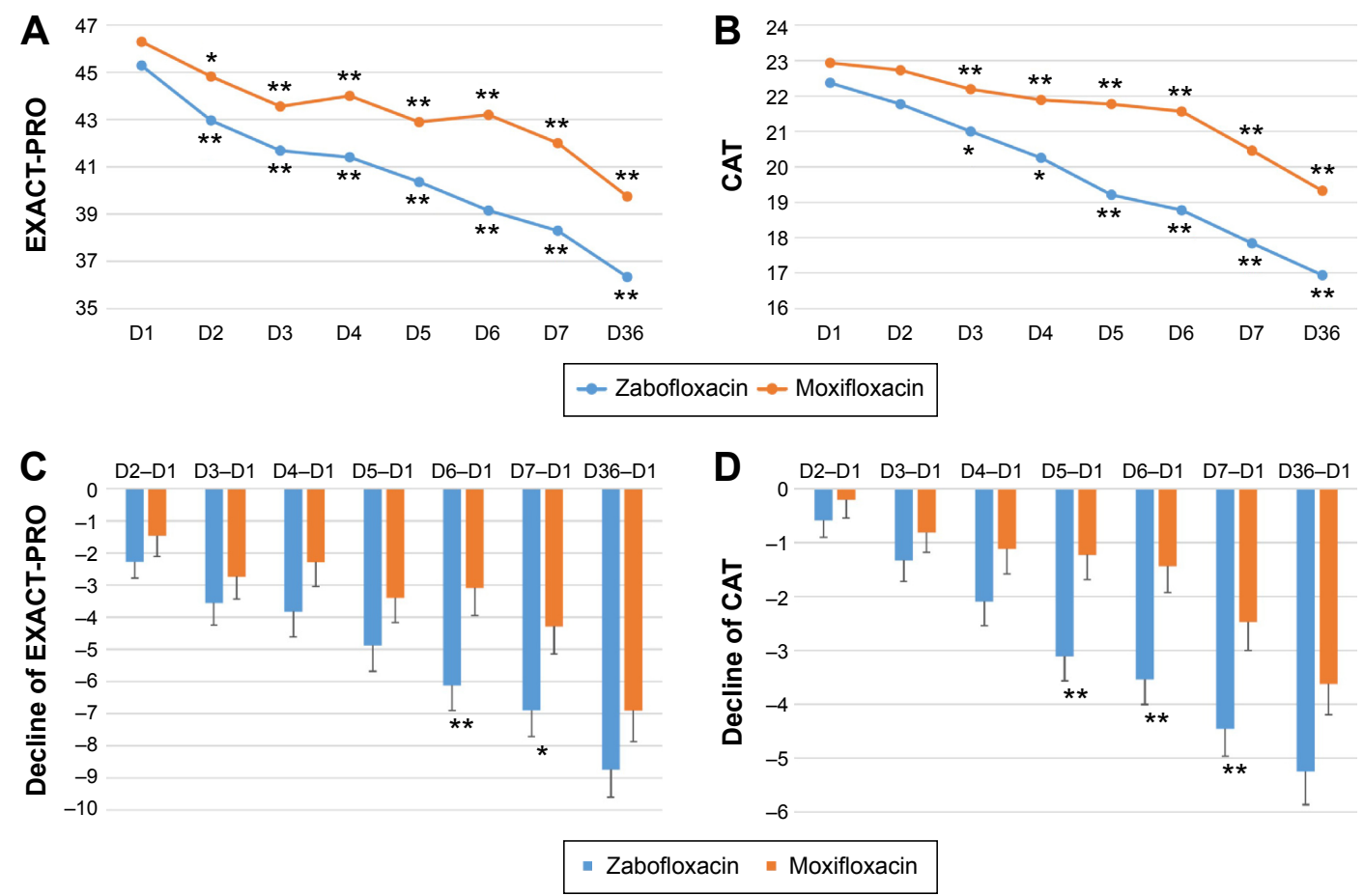

Figure 2 Clinical per protocol analysis of changes in the EXACT-PRO and CAT scores.

Notes: The EXACT-PRO $(\mathbf{A})$ and CAT scores $(\mathbf{B})$ decreased significantly compared with those on $\mathrm{DI}(* \mathrm{P}<0.05$, $* * P<0.0 \mathrm{I}$ vs $\mathrm{DI})$ in both the zabofloxacin and moxifloxacin groups. The decline in the EXACT-PRO (C) scores on D6 and D7 and the decline in the CAT scores (D) on D5, D6, and D7 were significantly greater in the zabofloxacin than in the moxifloxacin group ( $* P<0.05$ and $* * P<0.0$ I between the zabofloxacin and moxifloxacin groups).

Abbreviations: EXACT-PRO, EXAcerbations of Chronic Pulmonary Disease Tool-Patient-Reported Outcome; CAT, chronic obstructive pulmonary disease assessment test; D, day.
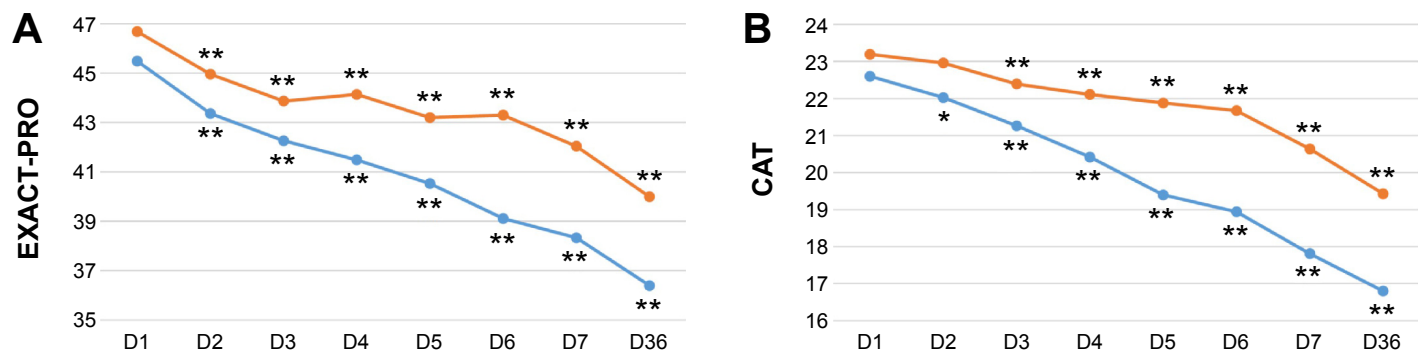

$\rightarrow$ Zabofloxacin $\rightarrow$ Moxifloxacin
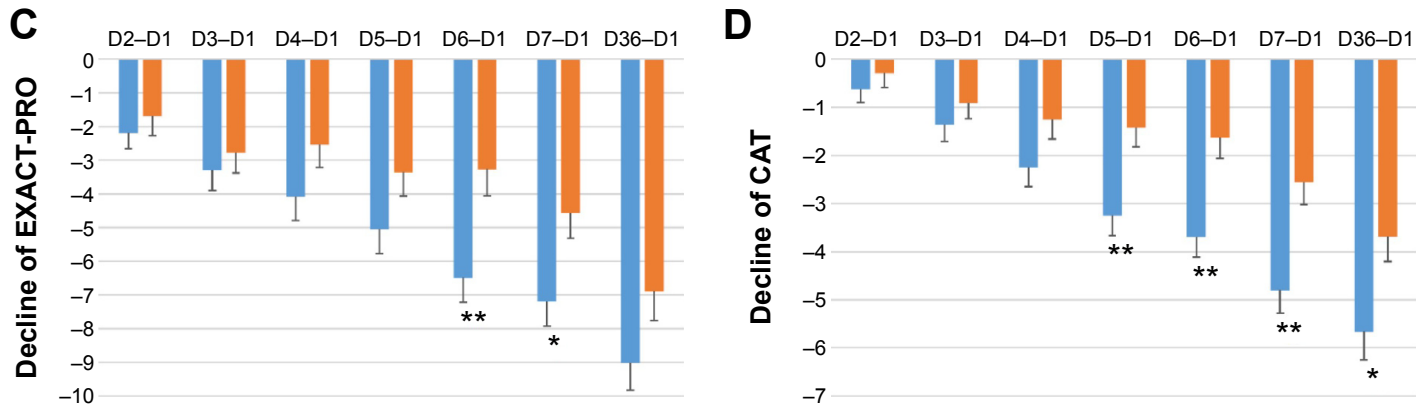

- Zabofloxacin = Moxifloxacin

Figure 3 Clinical intention-to-treat analysis of the changes of EXACT-PRO and CAT scores.

Notes: The EXACT-PRO $(\mathbf{A})$ and CAT scores $(\mathbf{B})$ decreased significantly compared with those on $\mathrm{DI}(* \mathrm{P}<0.05$, $* * P<0.0 \mathrm{I}$ vs $\mathrm{DI})$ in both the zabofloxacin and moxifloxacin groups. The decline in the EXACT-PRO (C) scores on D6 and D7 and the decline in the CAT scores (D) on D5, D6, D7, and D36 were significantly greater in the zabofloxacin than in the moxifloxacin group ( $* P<0.05$ and $* * P<0.01$ between the zabofloxacin and moxifloxacin groups).

Abbreviations: EXACT-PRO, EXAcerbations of Chronic Pulmonary Disease Tool-Patient-Reported Outcome; CAT, chronic obstructive pulmonary disease assessment test; D, day. 
Table 5 Adverse drug reactions

\begin{tabular}{|c|c|c|}
\hline \multirow[t]{2}{*}{ Adverse drug reaction } & \multicolumn{2}{|c|}{ Number (\%) of cases } \\
\hline & $\begin{array}{l}\text { Zabofloxacin } \\
n=175(\%)\end{array}$ & $\begin{array}{l}\text { Moxifloxacin } \\
n=167(\%)\end{array}$ \\
\hline \multicolumn{3}{|l|}{ Gastrointestinal disorder } \\
\hline Nausea & $3(1.7)$ & $4(2.4)$ \\
\hline Dry mouth & $0(0.0)$ & $\mathrm{I}(0.6)$ \\
\hline Diarrhea & $4(2.3)$ & $\mathrm{I}(0.6)$ \\
\hline Dyspepsia & I (0.6) & $0(0.0)$ \\
\hline Vomiting & I (0.6) & $0(0.0)$ \\
\hline Abdominal discomfort & $\mathrm{I}(0.6)$ & $0(0.0)$ \\
\hline Abdominal pain & $0(0.0)$ & I (0.6) \\
\hline Epigastric discomfort & $0(0.0)$ & $\mathrm{I}(0.6)$ \\
\hline Stomatitis & $0(0.0)$ & I (0.6) \\
\hline \multicolumn{3}{|c|}{ Respiratory, thoracic, and mediastinal problems } \\
\hline Chronic obstructive & I (0.6) & $0(0.0)$ \\
\hline \multicolumn{3}{|l|}{ pulmonary disease } \\
\hline Sputum retention & $0(0.0)$ & $\mathrm{I}(0.6)$ \\
\hline \multicolumn{3}{|l|}{ Nervous system disorders } \\
\hline Dizziness & $3(1.7)$ & I $(0.6)$ \\
\hline Headache & I (0.6) & I (0.6) \\
\hline Tremor & $0(0.0)$ & $\mathrm{I}(0.6)$ \\
\hline Paresthesia & $\mathrm{I}(0.6)$ & $0(0.0)$ \\
\hline Somnolence & $0(0.0)$ & $\mathrm{I}(0.6)$ \\
\hline \multicolumn{3}{|c|}{ General disorders and administration site conditions } \\
\hline Chest discomfort & $2(1.1)$ & $0(0.0)$ \\
\hline Face edema & $\mathrm{I}(0.6)$ & $0(0.0)$ \\
\hline Fatigue & $0(0.0)$ & $\mathrm{I}(0.6)$ \\
\hline Peripheral edema & $0(0.0)$ & $\mathrm{I}(0.6)$ \\
\hline \multicolumn{3}{|l|}{ Infections and infestations } \\
\hline Nasopharyngitis & $0(0.0)$ & $\mathrm{I}(0.6)$ \\
\hline \multicolumn{3}{|l|}{ Investigations } \\
\hline Glucose urine present & I $(0.6)$ & $0(0.0)$ \\
\hline $\begin{array}{l}\text { Red blood cell } \\
\text { microcytes present }\end{array}$ & $0(0.0)$ & $\mathrm{I}(0.6)$ \\
\hline \multicolumn{3}{|c|}{ Skin and subcutaneous tissue disorders } \\
\hline Pruritus & $\mathrm{I}(0.6)$ & $\mathrm{I}(0.6)$ \\
\hline Rash & I $(0.6)$ & $0(0.0)$ \\
\hline Drug eruption & $0(0.0)$ & $\mathrm{I}(0.6)$ \\
\hline Erythema & $\mathrm{I}(0.6)$ & $0(0.0)$ \\
\hline \multicolumn{3}{|l|}{ Cardiac disorders } \\
\hline Palpitations & $\mathrm{I}(0.6)$ & $0(0.0)$ \\
\hline \multicolumn{3}{|l|}{ Renal and urinary disorders } \\
\hline Hematuria & $0(0.0)$ & $\mathrm{I}(0.6)$ \\
\hline \multicolumn{3}{|c|}{ Musculoskeletal and connective tissue disorders } \\
\hline Flank pain & I (0.6) & $0(0.0)$ \\
\hline \multicolumn{3}{|l|}{ Eye disorders } \\
\hline Vision blurred & $0(0.0)$ & I (0.6) \\
\hline \multicolumn{3}{|l|}{ Vascular disorders } \\
\hline Flushing & $0(0.0)$ & I (0.6) \\
\hline Total & $17 / 175(9.7)$ & $16 / 167(9.6)$ \\
\hline
\end{tabular}

efficacy. Since a previous study ${ }^{8}$ compared a 5-day course of study drug (moxifloxacin) with a 7-day course of reference drug (levofloxacin), we also performed this study in a similar manner. In this study, in spite of the shorter period of drug administration, zabofloxacin showed comparable clinical effect on COPD exacerbation. Moreover, in spite of the shorter period of drug administration, symptom improvement was significantly faster in the zabofloxacin group.

This study is interesting when compared with the previous studies in that we focused on COPD rather than chronic bronchitis. Thus, this study can provide evidence that zabofloxacin is suitable for the treatment of patients with an acute exacerbation of COPD. Moreover, we stratified patients according to the type of chronic bronchitis. Subgroup analysis revealed that the efficacy of zabofloxacin was comparable with that of moxifloxacin in patients with and without chronic bronchitis. Thus, zabofloxacin can be used to treat patients with COPD exacerbation regardless of whether a patient has the phenotype of chronic bronchitis or not.

There are no clear guidelines regarding the period of antibiotic therapy for acute COPD exacerbations. The present study is valuable in that it is the first to show that a 5-day course of zabofloxacin yields a good clinical response to COPD exacerbations. PP analysis revealed that the clinical cure rate at the time of the TOC visit was $86.7 \%$. Considering that the study was limited to patients with moderate exacerbation (ie, patients who did not require hospitalization), this favorable result may be expected. However, the results provide valuable information regarding the period of antibiotic therapy required to manage COPD exacerbations.

To the best of our knowledge, this is the first study to measure EXACT-PRO and CAT scores for COPD patients during and after treatment with antibiotics. Interestingly, the results showed that zabofloxacin may be better than moxifloxacin in terms of symptom reduction during the treatment of exacerbations. The reason for this remains unclear. Further studies of CAT and EXACT-PRO scores during antibiotic treatment of exacerbations are needed to validate and support our results. However, we cautiously suggest that zabofloxacin may be more favorable than moxifloxacin. This is supported by other previous studies. For example, Kosowska-Shick et al ${ }^{6}$ showed that compared with other quinolones (including moxifloxacin), zabofloxacin was the most effective against both quinolone-susceptible and -resistant pneumococci. Park et $\mathrm{al}^{4}$ also showed that zabofloxacin was the most effective quinolone antibiotic against infections caused by Streptococcus pneumoniae. Zabofloxacin also shows rapid bactericidal activity and a long post-antibiotic effect against S. pneumoniae. ${ }^{16}$ This efficacy against $S$. pneumoniae can be explained by the finding that that DNA gyrase and DNA topoisomerase IV in S. pneumoniae are both targets for zabofloxacin. ${ }^{17}$ Zabofloxacin also shows good activity against atypical bacterial respiratory pathogens. ${ }^{18}$ Taken together, 
these results suggest that zabofloxacin has good clinical efficacy against respiratory pathogens. A Phase II, double-blind, multicenter study showed that zabofloxacin was comparable with moxifloxacin in terms of efficacy against communityacquired bacterial pneumonia. ${ }^{19}$

Zabofloxacin and moxifloxacin showed similar adverse event profiles in the present study. Gastrointestinal problems, including nausea and diarrhea, were the most common drugrelated adverse events. There were no serious adverse events that were related to both drugs. Both drugs were generally well tolerated, and few patients (none in the zabofloxacin group and three in the moxifloxacin group) dropped out due to adverse events. This result is compatible with that of a previous study comparing moxifloxacin with levofloxacin. ${ }^{8}$ Although gastrointestinal problems were the most common adverse events in that study, the discontinuation rate was also very low (approximately $2 \%$ ).

The present study has several limitations. First, although this was a randomized trial, there were some differences in baseline characteristics. Male sex and smoking status are both important factors in the management of COPD. We are unclear as to whether these two unmatched factors affected the clinical outcome. Second, the study was limited to patients suffering moderate exacerbations. Thus, the efficacy of zabofloxacin (orally for 5 days) applied only to this population. It is unclear whether such a protocol will be effective in hospitalized COPD patients with severe exacerbations. Further studies should examine the efficacy of zabofloxacin in hospitalized patients due to severe exacerbations. Third, 7 days of moxifloxacin instead of five may have affected the adverse effect of moxifloxacin. Fourth, bacteriologic eradication in this study was lower than two previous studies. ${ }^{8,9}$ We do not know exact reason for this difference. However, it may be because of the difference in the characteristics of the enrolled patients. In the previous two studies, enrolled patients were mainly suffering from chronic bronchitis while in this study they were COPD patients. Thus, baseline severity of disease may have resulted in a lower bacteriologic response. In our study, the mean forced expiratory volume in 1 second of patients was about $50 \%$.

\section{Conclusion}

Oral zabofloxacin (367 mg once daily for 5 days) was not inferior to oral moxifloxacin (400 mg once daily for 7 days) for the treatment of patients with COPD exacerbations. This treatment protocol may be a useful option for the treatment of COPD patients with exacerbations of moderate severity in the outpatient setting.

\section{Acknowledgments}

This study was supported by Dongwha Pharm. Co., Ltd., Seoul, Korea.

The members (other than authors) of this study are as follows: Sook Young Lee (Seoul St. Mary's Hospital, Seoul, Korea), Sung Hwan Jung, Jeong Woong Park (Gachon University Gil Medical Center, Incheon, Korea), Ju Sang Kim, Joong Hyun Ahn, Ah Young Shin, Hwa Young Lee (Incheon St. Mary's Hospital, Incheon, Korea), Woo Jin Kim, Yoonki Hong (Kangwon National University Hospital, Chencheon, Korea), Jong Deog Lee, Yi Young Jung (Gyeongsang National University, Jinju, Korea), Hee Joung Kim (Konkuk University Medical Center, Seoul, Korea), Chi Young Jung, Young June Jeon (Keimyung University Dongsan Medical Center, Daegu, Korea), Sang Yeub Lee, Kwang Ho In, Eun Joo Lee (Korea University Anam Hospital, Seoul, Korea), Sang-Do Lee, Sei Won Lee, Jae Seung Lee (Asan Medical Center, Seoul, Korea), Hun Gyu Hwang, Gune-Il Lim, Myung Shin Kim (Soonchunhyang University Hospital Gumi, Gumi, Korea), Ju Ock Na, Yong Hoon Kim, Jae Sung Choi, Ho Sung Lee (Soonchunhyang University Cheonan Hospital, Cheonan, Korea), Seung Soo Sheen, Keu Sung Lee (Ajou University Hospital, Suwon, Korea), Seung Won Ra, Kwang Won Seo, Yangjin Jegal, Jong-Joon Ahn (Ulsan University Hospital, Ulsan, Korea), Won Yeon Lee, Myoung Kyu Lee, Suk Joong Yong, Kye Chul Shin (Yonsei University Wonju Severance Christian Hospital, Wonju, Korea), Jin Hwa Lee, Yon Ju Ryu, Seok Jeong Lee (Ewha Womans University Mokdong Hospital, Seoul, Korea), Hang Jea Jang (Haeundae Paik Hospital, Busan, Korea), Yong Chul Lee, So Ri Kim, Seung Yong Park, Chi Ryang Chung (Chonbuk National University Hospital, Jeonju, Korea), Sung Soo Jung, Ju Ock Kim, Jeong Eun Lee (Chungnam National University Hospital, Daejeon, Korea), Kang Hyeon Choe, Ki Man Lee, Jin Young An (Chungbuk National University Hospital, Cheongju, Korea), Yong Bum Park, Changhwan Kim, Jae Young Lee, Eun Kyung Mo (Hallym University Kangdong Sacred Heart Hospital, Seoul, Korea), Seung Hun Jang, Ki Suck Jung (Hallym University Sacred Heart Hospital, Anyang, Korea), Tae-Hyung Kim, Ji-Young Moon (Hanyang University Guri Hospital, Guri, Korea), Hye Sook Choi (Dongguk University Gyeongju Hospital, Gyeongju, Korea), Young Sam Kim (Severance Hospital, Seoul, Korea), Kyeong-Cheol Shin (Yeungnam University Medical Center, 
Daegu, Korea), Sung Ho Yoon (Chosun University Hospital, Gwangju, Korea).

We express our special thanks to Cheol Hee Lim and Sun Hea Lee, Dongwha Pharm. Co., Ltd., Seoul, Korea.

\section{Disclosure}

CK Rhee received consulting/lecture fees from MSD Korea, AstraZeneca Korea, Novartis Korea, GSK Korea, Takeda Korea, Mundipharma Korea, Sandoz Korea and Boehringer-Ingelheim Korea. YM Oh received honoraria/ consulting fees from DongWha, MSD Korea, AstraZeneca Korea, GlaxoSmithKline Korea, Novartis, and BoehringerIngelheim Korea. The other authors report no other conflicts of interest in this work.

\section{References}

1. Pela R, Marchesani F, Agostinelli C, et al. Airways microbial flora in COPD patients in stable clinical conditions and during exacerbations: a bronchoscopic investigation. Monaldi Arch Chest Dis. 1998; 53(3):262-267.

2. Sethi S, Murphy TF. Infection in the pathogenesis and course of chronic obstructive pulmonary disease. $N$ Engl J Med. 2008;359(22): 2355-2365.

3. Wedzicha JA, Singh R, Mackay AJ. Acute COPD exacerbations. Clin Chest Med. 2014;35(1):157-163.

4. Park HS, Kim HJ, Seol MJ, Choi DR, Choi EC, Kwak JH. In vitro and in vivo antibacterial activities of DW-224a, a new fluoronaphthyridone. Antimicrob Agents Chemother. 2006;50(6):2261-2264.

5. Kwon AR, Min YH, Ryu JM, Choi DR, Shim MJ, Choi EC. In vitro and in vivo activities of DW-224a, a novel fluoroquinolone antibiotic agent. J Antimicrob Chemother. 2006;58(3):684-688.

6. Kosowska-Shick K, Credito K, Pankuch GA, et al. Antipneumococcal activity of DW-224a, a new quinolone, compared to those of eight other agents. Antimicrob Agents Chemother. 2006;50(6):2064-2071.

7. Petitpretz P, Chone C, Tremolieres F. Levofloxacin $500 \mathrm{mg}$ once daily versus cefuroxime $250 \mathrm{mg}$ twice daily in patients with acute exacerbations of chronic obstructive bronchitis: clinical efficacy and exacerbationfree interval. Int J Antimicrob Agents. 2007;30(1):52-59.

8. Urueta-Robledo J, Ariza H, Jardim JR, et al. Moxifloxacin versus levofloxacin against acute exacerbations of chronic bronchitis: the Latin American Cohort. Respir Med. 2006;100(9):1504-1511.
9. Chodosh S, DeAbate CA, Haverstock D, Aneiro L, Church D. Shortcourse moxifloxacin therapy for treatment of acute bacterial exacerbations of chronic bronchitis. The Bronchitis Study Group. Respir Med. 2000;94(1):18-27.

10. Wilson R, Kubin R, Ballin I, et al. Five day moxifloxacin therapy compared with 7 day clarithromycin therapy for the treatment of acute exacerbations of chronic bronchitis. $J$ Antimicrob Chemother. 1999;44(4):501-513.

11. DeAbate CA, Mathew CP, Warner JH, Heyd A, Church D. The safety and efficacy of short course (5-day) moxifloxacin vs azithromycin in the treatment of patients with acute exacerbation of chronic bronchitis. Respir Med. 2000;94(11):1029-1037.

12. Zervos M, Martinez FJ, Amsden GW, Rothermel CD, Treadway G. Efficacy and safety of 3-day azithromycin versus 5-day moxifloxacin for the treatment of acute bacterial exacerbations of chronic bronchitis. Int J Antimicrob Agents. 2007;29(1):56-61.

13. Grassi C, Casali L, Curti E, Tellarini M, Lazzaro C, Schito G. Efficacy and safety of short course (5-day) moxifloxacin vs 7-day ceftriaxone in the treatment of acute exacerbations of chronic bronchitis (AECB). J Chemother. 2002;14(6):597-608.

14. Starakis I, Gogos CA, Bassaris H. Five-day moxifloxacin therapy compared with 7-day co-amoxiclav therapy for the treatment of acute exacerbation of chronic bronchitis. Int J Antimicrob Agents. 2004; 23(2):129-137.

15. Liu KX, Xu B, Wang J, et al. Efficacy and safety of moxifloxacin in acute exacerbations of chronic bronchitis and COPD: a systematic review and meta-analysis. $J$ Thorac Dis. 2014;6(3):221-229.

16. Kim HJ, Seol MJ, Park HS, et al. Antimicrobial activity of DW-224a, a new fluoroquinolone, against Streptococcus pneumoniae. J Antimicrob Chemother. 2006;57(6):1256-1258.

17. Park HS, Jung SJ, Kwak JH, Choi DR, Choi EC. DNA gyrase and topoisomerase IV are dual targets of zabofloxacin in Streptococcus pneumoniae. Int J Antimicrob Agents. 2010;36(1):97-98.

18. Williams L, Shackcloth J, Jung YH, Morrissey I. Activity of DW-224a and comparators against atypical bacterial respiratory pathogens. Paper presented at: Interscience Conference on Antimicrobial Agents and Chemotherapy; October 30; 2004; Washington, DC.

19. Kim YS, Kim MJ, Back KR, et al. A phase 2, multi-dose, double-blind, randomized, multicenter, safety and efficacy study of zabofloxacin vs moxifloxacin in the treatment of mild to moderate community-acquired pneumonia. Paper presented at: Interscience Conference on Antimicrobial Agents and Chemotherapy; September 9; 2012; San Francisco, CA.
International Journal of COPD

\section{Publish your work in this journal}

The International Journal of COPD is an international, peer-reviewed journal of therapeutics and pharmacology focusing on concise rapid reporting of clinical studies and reviews in COPD. Special focus is given to the pathophysiological processes underlying the disease, intervention programs, patient focused education, and self management protocols.

\section{Dovepress}

This journal is indexed on PubMed Central, MedLine and CAS. The manuscript management system is completely online and includes a very quick and fair peer-review system, which is all easy to use. Visit http://www.dovepress.com/testimonials.php to read real quotes from published authors. 